\title{
In Vitro Biotransformation, Safety, and Chemopreventive Action of Novel 8-Methoxy-Purine-2,6-Dione Derivatives
}

\author{
Malgorzata Anna Marć ${ }^{1}$. \\ Enrique Domínguez-Álvarez ${ }^{1,2} \cdot$ Karolina Stoczyńska $^{1}$ • \\ Pawel Żmudzki ${ }^{3}$ - Grażyna Chłoń-Rzepa ${ }^{3}$. \\ Elżbieta Pękala ${ }^{1}$ (D)
}

Received: 6 April 2017 / Accepted: 29 May 2017 /

Published online: 17 June 2017

(C) The Author(s) 2017. This article is an open access publication

\begin{abstract}
Metabolic stability, mutagenicity, antimutagenicity, and the ability to scavenge free radicals of four novel 8-methoxy-purine-2,6-dione derivatives (compounds 1-4) demonstrating analgesic and anti-inflammatory properties were determined. Metabolic stability was evaluated in Cunninghamella and microsomal models, mutagenic and antimutagenic properties were assessed using the Ames and the Vibrio harveyi tests, and free radical scavenging activity was evaluated with 2,2-diphenyl-1-picrylhydrazyl radical scavenging assay. In the Cunninghamella model, compound 2 did not undergo any biotransformation; whereas 3 and 4 showed less metabolic stability: 1-9 and 53$88 \%$ of the parental compound, respectively, underwent biotransformation reactions in different Cunninghamella strains. The metabolites detected after the biotransformation of 3 and 4 were aromatic hydroxylation and $N$-dealkylation products. On the other hand, the $N$-dealkylation product was the only metabolite formed in microsome assay. Additionally, these derivatives do not possess mutagenic potential in microbiological models (Vibrio harveyi and Salmonella typhimurium) considered. Moreover, all compounds showed a strong chemopreventive activity in the modified Vibrio harveyi strains BB7X and BB7M. However, radical scavenging activity was not the mechanism which explained the observed chemopreventive activity.
\end{abstract}

Elżbieta Pękala

elzbieta.pekala@uj.edu.pl

1 Department of Pharmaceutical Biochemistry, Faculty of Pharmacy, Jagiellonian University Medical College, 9 Medyczna Street, 30-688 Kraków, Poland

2 Institute of General Organic Chemistry, Spanish National Research Council (IQOG-CSIC), Juan de la Cierva 3, 28006 Madrid, Spain

3 Department of Medicinal Chemistry, Faculty of Pharmacy, Jagiellonian University Medical College, 9 Medyczna Street, 30-688 Kraków, Poland 
Keywords Alternative test - Ames test - Cunninghamella assay - DPPH assay - Microsomal stability

\section{Introduction}

During the biotransformation processes, drugs and chemicals are structurally modified by various enzymatic systems to form more polar substances, which can be excreted more easily than the original compounds. Problems arise when these modifications generate toxic products $[1,2]$. Traditionally, drug metabolism studies use in vivo experiments in mice, rat or guinea pig, or chimeric mouse models with transplanted human hepatocytes [3, 4]. However, these models can create ethical dilemmas; and the experiments are expensive and time consuming [3]. In addition, metabolites are sometimes produced in low amounts, thus hindering their identification [4].

Enzymatic systems involved in the metabolism of exogenous organic compounds are similar in mammals and in certain fungal microorganisms, like Cunninghamella. Therefore, Cunninghamella fungi can be used as an alternative to in vivo metabolism models [1-3, 5-8]. The use of these microorganisms enables the reduction of research costs and does not arise ethical dilemmas. These alternative tests are simple and reliable as metabolites can be easily extracted to be identified later using instrumental analytical techniques [3]. Cunninghamella echinulata contains a rich set of microsomal cytochrome P450, and it can conduct phase I (oxidative) and phase II (conjugative) biotransformation under certain conditions. Moreover, the fungus possesses the ability to metabolize a wide variety of xenobiotics in regio- and stereo-selective manners [9].

A second alternative to in vivo metabolism assays of chemical compounds are in vitro screening assays with liver microsomes: they can predict the metabolic routes of tested compounds, as well as kinetics of these transformations [10]. Cytochrome P450 enzymes are involved in phase I reactions, whereas phase II reactions are commonly carried out by different transferase enzymes such as glutathione transferase and $N$-acetyltransferase [11]. As microsomal assays have a good reproducibility and performing them is straightforward, they are becoming a standard in vitro screening in drug discovery processes [12-14]. Finally, liver microsomes from different species (such as rat, mouse, dog, monkey, and human) can be used to provide more accurate data [11].

Zygmunt et al. $[15,16]$ reported in previous studies that some novel derivatives of 8methoxy-purine-2,6-diones substituted at 7-position of purine core by carboxylic, ester, or amide moieties showed potent anti-inflammatory and analgesic activity, superior to the reference drug, in preliminary pharmacological studies $[15,16]$. Their analgesic properties were evaluated in two pharmacological in vivo models: formalin and writhing syndrome tests. On the other hand, anti-inflammatory potential of the aforesaid compounds was determined using zymosan-induced peritonitis and carrageenan-induced edema models. Derivatives containing an amide substituent showed very strong analgesic and anti-inflammatory (antiedematous) activities. The most active compound (4) showed a 23-fold and a 36-fold higher activity than acetylsalicylic acid (the analgesic used in clinical practice considered as reference) in the writhing syndrome test in mice and in the formalin test in mice, respectively $[15,16]$. Thus, these newly synthesized derivatives represent a new class of analgesic and antiinflammatory agents with possible future therapeutic applications in the treatment of inflammatory diseases and in the attenuation of pain. However, once verified their promising activity, 
more in-depth studies need to be performed in their pharmacology, pharmacokinetics, safety, and toxicology, among other fields, to evaluate the feasibility of the possible clinical applications of these compounds, as well as to know better how they exert their action.

In line to this, the aim of this study was to investigate in vitro biotransformation and some biological activities of the four (1-4) most promising 8-methoxy-purine-2,6-dione derivatives (Fig. 1) selected on the basis of their activity $[15,16]$. The activities evaluated in the present study are mutagenicity, antimutagenicity, metabolic biotransformations, and the ability of the compounds to scavenge free radicals. Among the four compounds tested, there are 2-(8methoxy-1,3-dimethyl-2,6-dioxo-purin-7-yl)acetic acid (1), methyl 2-(8-methoxy-1,3-dimethyl-2,6-dioxo-purin-7-yl)acetate (2), $N$-benzyl-4-(8-methoxy-1,3-dimethyl-2,6-dioxo-purin-7yl)butanamide (3), and 8-methoxy-1,3-dimethyl-7-[4-oxo-4-(4-phenylpiperazin-1yl)butyl]purine-2,6-dione (4).

\section{Materials and Methods}

\section{Tested Compounds}

Four 8-methoxy-purine-2,6-dione derivatives (1-4) described in previous publications [15, 16] were provided by Dr. Grażyna Chłon-Rzepa from the Department of Medicinal Chemistry, Jagiellonian University Medical College. The structures of these compounds were established on the basis of CHN elemental analysis and spectral data (IR, ${ }^{1} \mathrm{HNMR}$, and mass spectra). Additionally, qualitative analysis was performed using TLC and HPLC.

In the Cunninghamella biotransformation assay, $12.5 \mathrm{mg}$ of compounds $2-4$ were dissolved in $0.5 \mathrm{~mL}$ of DMSO to be later inoculated in $24.5 \mathrm{~mL}$ of broth medium containing fungus, being thus $0.5 \mathrm{mg} / \mathrm{mL}$ the final concentration. For rat microsomal assay, $5.1 \mathrm{mM}$ stock solutions of compounds 1 and 3 were prepared in water and ethanol, respectively. From them, $0.2 \mathrm{mM}$ working solutions were prepared by dilution with water. In mutagenic tests, compounds $1-4$ were dissolved in pure DMSO to obtain the corresponding stock solution $(10 \mathrm{mg} /$ $\mathrm{mL}$ ). Working solutions were prepared by 1:100 dilution of stock solution in water, and final compound concentration assayed was $40 \mathrm{ng} / \mathrm{mL}$. Finally, for 2,2-diphenyl-1-picrylhydrazyl (DPPH) assay, $1 \mathrm{mM}$ stock solutions of compounds 1-4 were prepared in methanol.

1<smiles>COc1nc2c(c(=O)n(C)c(=O)n2C)n1CC(=O)O</smiles><smiles>[Z]Oc1nc2c(c(=O)n(C)c(=O)n2C)n1CC(=O)OC</smiles>

3<smiles>COc1nc2c(c(=O)n(C)c(=O)n2C)n1CCCC(=O)NCc1ccccc1</smiles><smiles>COc1nc2c(c(=O)n(C)c(=O)n2C)n1CCCC(=O)N1CCN(c2ccccc2)CC1</smiles>

Fig. 1 Chemical structures of compounds 1-4 


\section{Reagents}

4-Nitroquinoline $N$-oxide (NQNO), DMSO, L-histidine monochloride, yeast extract, levallorphan, $\mathrm{NADP}^{+}$, glucose-6-phosphate sodium salt, glucose-6-phosphate dehydrogenase, potassium hydroxide, DPPH, gallic acid, and ascorbic acid were purchased from SigmaAldrich (Seelze, Germany); Nutrient Broth No. 2, from Argenta (Poznań, Poland); glycerol and neomycin sulfate, from Pharma Cosmetic (Kraków, Poland); sodium sulfate, sodium chloride, D-glucose, and dipotassium hydrogen phosphate from Chempur (Piekary Śląskie, Poland); dichloromethane from Stanlab (Lublin, Poland); and agar-agar, bacto-peptone, beef extract, potato dextrose agar, and 0.2-mm silica-coated aluminum TLC plates from Merck (Darmstadt, Germany).

\section{Cunninghamella Strains and Culture Conditions}

Three different strains of Cunninghamella were used: Cunninghamella echinulata NRRL 1384, Cunninghamella blakesleeana 1908 DMS, and Cunninghamella elegans 1906 DMS. The first was provided by Dr. A.J. Carnell (University of Liverpool, UK), whereas the two remaining were purchased from Deutsche Sammlung von Mikroorganismen und Zellkulturen GmbH (DMSZ, Braunschweig, Germany).

Cunninghamella strains were cultivated on solid potato dextrose agar (PDA) plates at $28{ }^{\circ} \mathrm{C}$. After 4 days, a liquid fermentation basal medium was prepared by the addition of $6 \mathrm{~g}$ of D-glucose, $1.5 \mathrm{~g}$ of NaCl, $1.5 \mathrm{~g}$ of yeast extract from Saccharomyces cerevisiae, and $1.5 \mathrm{~g}$ of $\mathrm{K}_{2} \mathrm{HPO}_{4}$ to $300 \mathrm{~mL}$ of distilled water. Aliquots of $24.5 \mathrm{~mL}$ of medium were inoculated with $300 \mu \mathrm{L}$ of suspension of fungal spores obtained after wetting colonies in PDA. The fungi were incubated with shaking until the formation of spherical clumps.

\section{Cunninghamella Biotransformation Assay}

Compounds 2-4 were evaluated in this experiment. Once microorganisms formed spherical clumps in the preliminary incubation, the corresponding tested compound was added to the broth and the mixture was incubated for 7 days. Two control experiments were also performed, in the absence of compound and fungus, respectively [3, 9]. Biotransformation progress was monitored by TLC and by liquid chromatography electrospray ionization-tandem mass spectrometry (LC-MS/MS). Monitoring was performed at four different incubation times: $30 \mathrm{~min}$, 3 days, 5 days, and 7 days.

To analyze metabolites in LC-MS/MS, an aliquot of $500 \mu \mathrm{L}$ of filtered sample was taken and extracted with $500 \mu \mathrm{L}$ of dichloromethane. The organic fraction was dried with sodium sulfate, filtered, and evaporated. The obtained residue was analyzed in LC-MS/MS. At the end of experiment, all the remaining medium was extracted with $25 \mathrm{~mL}$ of dichloromethane after separating the fungal clumps by filtration over cellulose filters $[3,17]$.

\section{Microsomal Stability Assay}

Compounds 1 and 3 were studied in a biotransformation assay using rat liver microsomes following procedures described previously [18-22]. The reagents used in this assay were levallorphan (internal standard) and NADPH-regenerating system, which contained phosphate buffer ( $\mathrm{pH}$ 7.4), NADP ${ }^{+}$, glucose-6-phosphate, and glucose-6-phosphate dehydrogenase [18]. 
The final protein concentration in the assay was $0.4 \mathrm{mg} / \mathrm{mL}$. Compounds 1 and 3 were tested at a final concentration of $20 \mu \mathrm{M}$.

The amount of a parental compound remaining in a solution after biotransformation was monitored using LC-MS/MS as described previously in "Cunninghamella Biotransformation Assay." Graphs representing ln of percentage of parent compound remaining versus incubation time were drawn to calculate in vitro half-time $\left(t_{1 / 2}\right)$ from the slope of linear regression. To obtain intrinsic clearance $\left(\mathrm{Cl}_{\text {int }}\right)$ of a tested compound in rat liver microsomal assay, $t_{1 / 2}$ previously calculated was substituted at the equation $\mathrm{Cl}_{\text {int }}=\left[\left(V_{\text {mic }} / P_{\text {mic }}\right) \times 0.693 / t_{1 / 2}\right]$, being $V_{\text {mic }}$ and $P_{\text {mic }}$ the volume of incubation in microliters and the amount of incubated protein in milligrams, respectively [23, 24].

\section{Analytical Methods}

TLC experiments were performed using 0.2-mm silica-coated aluminum plates, being a 95:5 dichloromethane/methanol mixture the mobile phase. The plates were observed under UV light.

Agilent 1100 HPLC system (Agilent Technologies, Waldbronn, Germany) equipped with a Xbridge ${ }^{\mathrm{TM}} \mathrm{C} 18$ analytical column $(2.1 \times 30 \mathrm{~mm}, 3.5 \mu \mathrm{M}$; Waters, Dublin, Ireland $)$ was used in the chromatographic separation of LC-MS/MS analysis. An elution gradient of acetonitrile and water, with $0.1 \%$ of formic acid, was used as a mobile phase. MS spectra were taken at Applied Biosystems MDS Sciex API 200 triple quadrupole mass analyzer (Concord, Ontario, Canada) with an electrospray ionization (ESI) interface between HPLC and MS.

\section{Bacterial Strains}

Four Vibrio harveyi strains were used in this study: a wild-type BB7 (naturally found in the Baltic Sea) and three genetically modified strains, namely BB7M (obtained by the introduction of pAB1273 plasmid containing the genes $m u c A$ and $m u c B$ to enhance the error-prone DNA repair), BB7X (hypersensitive to UV radiation after genetic modification with Tn5TpMCS), and BB7XM (containing the two abovementioned modifications) [25-28]. These strains were kindly provided by Prof. G. Wegrzyn (Department of Molecular Biology, University of Gdańsk, Poland).

In the Ames test, Salmonella typhimurium TA100 strain was used. This strain has a base-pair substitution that can be reverted by mutations at GC pairs. In addition, it contains mutations at $u v r B$-bio and $r f a$ genes to eliminate excision repair mechanisms and to make the bacteria more permeable to chemicals, respectively. Finally, TA100 also includes pKM101 plasmid, which enhances both chemical and UV-induced mutagenesis via an increase in the error-prone recombinational DNA repair pathway [29, 30]. TA100 strain of $S$. typhimurium was provided by Dr. T. Nohmi (Division of Genetics and Mutagenesis, National Institute of Hygienic Sciences, Tokyo, Japan). V. harveyi and S. typhimurium bacterial cultures were maintained at 30 and $37^{\circ} \mathrm{C}$, respectively.

\section{Mutagenic Agent}

A standard mutagen NQNO was used as a positive control in mutagenicity assays and as a mutagen added to all probes (except negative controls) in antimutagenicity tests. It causes 
point mutations at the genome as it induces $\mathrm{G}: \mathrm{C} \rightarrow \mathrm{A}: \mathrm{T}$ transitions in Vibrio harveyi strains [27, 31] and in other bacteria such as Escherichia coli and S. typhimurium [32]. NQNO was initially dissolved in DMSO. Next, its working solution was prepared in sterile water, being $40 \mathrm{ng} / \mathrm{mL}$ its final concentration.

\section{Vibrio harveyi and Ames Mutagenicity Assays}

To perform the $V$. harveyi mutagenicity test, $10 \mu \mathrm{L}$ of a working solution of the tested compound $(100 \mu \mathrm{g} / \mathrm{mL})$ were added to $V$. harveyi bacterial culture $\left(\mathrm{OD}_{600}=0.1\right)$ in $\mathrm{NaCl}$-containing BOSS liquid medium, being $40 \mathrm{ng} / \mathrm{mL}$ the final concentration of the tested compound. The samples were left incubating till $\mathrm{OD}_{600}$ increased to 0.3-0.4. Then, an inoculum containing $5 \times 10^{6}$ cells was added to solid BOSS agar plate supplemented with neomycin sulfate at a final concentration of $100 \mu \mathrm{g} / \mathrm{mL}$. Samples were left incubating $48 \mathrm{~h}$ at $30{ }^{\circ} \mathrm{C}$, and revertant colonies were counted manually. A positive control with NQNO and two negative controls (DMSO and blank water probe) were also tested. Each experiment was performed in triplicate, and results were given in terms of mutagenic index (MI), which is the quotient between the number of revertant colonies induced in a test sample and the number of revertants in a negative control [33-35]. A compound is considered mutagenic if MI is above 2.0 or if the number of revertants is higher in compound samples that in NQNO ones [26, 31].

Additionally, two different final concentrations of compounds (40 and $500 \mathrm{ng} / \mathrm{mL}$ ) were evaluated in the Ames test to confirm the results obtained in the Vibrio harveyi assay using a second independent method. Positive (NQNO, concentration of $40 \mathrm{ng} / \mathrm{mL}$ ) and negative (DMSO) controls were also tested. S. typhimurium was cultivated in a fresh medium $12 \mathrm{~h}$ prior to a test. To perform mutagenicity assay, $100 \mu \mathrm{L}$ of an overnight culture (containing approx. $1 \times 10^{8}$ to $2 \times 10^{8}$ bacteria) were inoculated along with $50 \mu \mathrm{L}$ of a tested compound in $2 \mathrm{~mL}$ of top agar supplemented with traces of histidine and of biotin. Then the mixture was added over the surface of GM agar plates and was left incubating $48 \mathrm{~h}$ at $37{ }^{\circ} \mathrm{C}$. Afterwards, the colonies were counted manually $[25,28]$. A compound is considered mutagenic if $\mathrm{MI}>2.0$ or if compound samples contain more revertants than NQNO controls [33-36].

\section{Vibrio harveyi Antimutagenicity Assay}

Procedure followed in the $V$. harveyi antimutagenicity assay [34, 35] was similar to the mutagenicity method described before. Differential point is that $10 \mu \mathrm{L}$ of NQNO (final concentration of $40 \mathrm{ng} / \mathrm{mL}$ ) were added to $V$. harveyi bacterial culture in BOSS liquid medium $15 \mathrm{~min}$ after the addition of tested compound to the culture. The results were expressed as the inhibition percentage of mutagenic effect, which was calculated with the formula: $100-\left[\left(R_{1} / R_{2}\right) \times 100\right]$, where $R_{1}$ is the number of revertants per plate in the presence of both mutagen and tested substance, and $R_{2}$ is the number of revertants per plate in the presence of mutagen $[34,36]$. The antimutagenic effect was considered weak/absent, moderate, or strong when the inhibition percentage was up to $25 \%$, from 25 to $40 \%$, and higher than $40 \%$, respectively [34, 37]. S. typhimurium antimutagenicity assay was based on similar assumptions as the $V$. harveyi antimutagenicity test. In this case, the chemopreventive activity was determined at two concentrations of a tested compounds and a standard mutagen, i.e., 40 and $500 \mathrm{ng} / \mathrm{mL}$. 


\section{DPPH Assay}

The capacity of selected 8-methoxy-purine-2,6-dione derivatives to scavenge stable free radical DPPH was assessed [22, 38-42]. A blank and a triplicate of 10 different concentrations of each compound or of each positive control (gallic and ascorbic acids) were evaluated in the experiment. Aforesaid concentrations of compounds were obtained by dilution from a $1000 \mu \mathrm{M}$ stock solution in methanol. One hundred microliters of DPPH stock solution were added to each well; later, $100 \mu \mathrm{L}$ of the corresponding compound dilution were added. Consequently, the final concentration of DPPH radical was $250 \mu \mathrm{M}$, whereas the final concentrations of the tested compounds or positive controls were 500, 375, 250, 125, 62.5, $50,37.5,25,12.5$, and $6.25 \mu \mathrm{M}$. Afterwards, the 96-plate was wrapped with aluminum and stored in the dark $30 \mathrm{~min}$ prior to the spectrophotometric measurement at a $517-\mathrm{nm}$ wavelength. The obtained absorbances were used to calculate the percentage of radical scavenged by the action of a compound or of a positive control at each concentration using a formula $100 \times\left(A_{0}-A_{\mathrm{m}}\right) / A_{0}$. In this equation $A_{\mathrm{m}}$ is the average absorbance of the three triplicates measured at the corresponding concentration and $A_{0}$ is the average absorbance of DPPH radical in the absence of the tested substance. Knowing inhibition data at the different concentrations, $\mathrm{IC}_{50}$ was calculated when applicable [40].

\section{Results}

\section{Cunninghamella Biotransformation}

The metabolic degradation of compounds 2-4 was studied in the Cunninghamella biotransformation assay, being these derivatives tested in three different Cunninghamella species: C. echinulata, C. blakesleeana, and C. elegans. Compound 1 was not evaluated within this experiment due to its structural similarity to compound 2. Metabolites were determined through the study of the corresponding LC-MS/MS spectra. Unless stated otherwise, the metabolites discussed onwards are the ones found at the end of experiment after 7-day incubation of investigated purine-2-6-dione derivatives with the corresponding Cunninghamella strain.

None of the three Cunninghamella strains evaluated generated detectable metabolites of 2 in a biotransformation assay, according to the LC-MS/MS spectra recorded. The main peak observed in LC-MS/MS corresponded with protonated molecule $[\mathrm{M}+\mathrm{H}]^{+}$of $2(\mathrm{~m} / \mathrm{z}, 283)$ and was eluted in $\mathrm{LC}$ at a retention time of $3.52 \mathrm{~min}$.

In case of 3, a protonated molecule $[\mathrm{M}+\mathrm{H}]^{+}(\mathrm{m} / \mathrm{z}, 386)$ of a parental compound (Fig. 2) remained to be the most important derivative ion present in the extract, as only a $0.6,4.6$, and $7.6 \%$ of a tested compound was metabolized at the end of experiment in C. elegans, C. blakesleeana, and C. echinulata, respectively (Table 1). The main metabolite (3-M4) in three strains corresponded to the product of aromatic hydroxylation (Fig. 3) of a parental compound, and was eluted at a retention time of $3.62 \mathrm{~min}(\mathrm{~m} / \mathrm{z} 402)$. The percentages of the metabolite observed were $0.6,4.6$, and $4.2 \%$ in C. elegans, C. blakesleeana, and C. echinulata, respectively. No more metabolites were detected in the first two strains. C. echinulata generated three additional metabolites in the biotransformation assay. The first of these three metabolites (3-M1) had a retention time of $2.75 \mathrm{~min}$, an abundance of $1.5 \%$, and an $\mathrm{m} / \mathrm{z}$ of 296. On the other hand, the main peak in MS of the two remaining metabolites had a molecular 


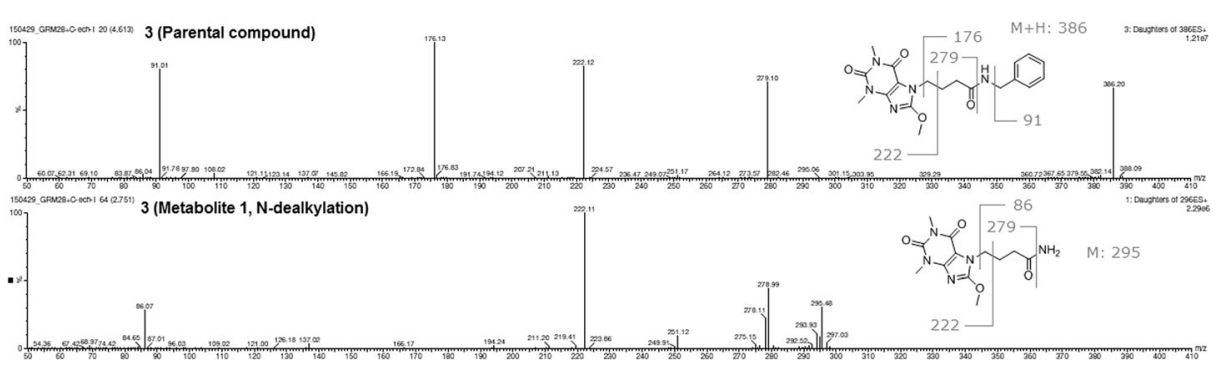

Fig. 2 LC-MS/MS spectra compound 3 and its 3-M1 metabolite

weight of 402 (Fig. 3). The percentages of appearance of these two metabolites and their retention times were $3.79 \mathrm{~min}$ and $0.3 \%$ for the first metabolite (3-M3) and $4.29 \mathrm{~min}$ and $1.6 \%$ for the second (3-M2) (Table 1, Figs. 3 and 4).

A protonated molecule $[\mathrm{M}+\mathrm{H}]$ at $\mathrm{m} / \mathrm{z}, 441$ was detected in mass spectra of four after the biotransformation processes, corresponding to a molecular weight $(\mathrm{m} / \mathrm{z} 440)$ of a parental compound. In all Cunninghamella strains, only one metabolite (4-M1), the product of aromatic hydroxylation, was observed, at a retention time of $3.37 \mathrm{~min}$ and with $\mathrm{m} / \mathrm{z} 457$. The calculated percentages of the parental compound metabolized in each case were 53.0,66.6, and $87.6 \%$ in C. elegans, C. blakesleeana, and C. echinulata, respectively (Table 1, Fig. 4).

\section{Microsomal Biotransformation}

The metabolic stability of compounds 1 and 3 was measured by the incubation with rat liver microsomes and subsequent monitoring of the metabolites formed by LC-MS/MS. Compound 2 was not examined with microsomes due to its metabolic stability in a former Cunninghamella assay. During the microsomal biotransformation, compound 1 was metabolically stable as no metabolites were observed after $1 \mathrm{~h}$ of its incubation with microsomes: only a protonated paternal compound was detected in LC-MS/MS spectra.

On the other hand, only one metabolite (3-M1) of compound 3 (Table 2) was observed after rat microsomal incubation, being its molecular weight and its retention time $296 \mathrm{~g} / \mathrm{mol}$ and $2.78 \mathrm{~min}$, respectively. The metabolite found was the product of the $\mathrm{N}$-dealkylation of a

Table 1 Metabolites of compounds 3 and 4 detected in Cunninghamella biotransformation assay

\begin{tabular}{|c|c|c|c|c|c|c|c|}
\hline \multicolumn{4}{|c|}{ Metabolite/compound } & \multicolumn{4}{|c|}{$\begin{array}{l}\% \text { of compound/metabolite in blank or different } \\
\text { Cunninghamella strains }\end{array}$} \\
\hline Cmp. & $\begin{array}{l}t_{\mathrm{r}} \\
(\min )\end{array}$ & $\begin{array}{l}{[\mathrm{M}+} \\
\mathrm{H}]^{+}\end{array}$ & $\begin{array}{l}\text { Biotransformation reaction } \\
\text { type }\end{array}$ & Blank & C. elegans & C. blakesleeana & C. echinulata \\
\hline 3 & 4.61 & 386 & Parental compound & 100.0 & 99.4 & 95.4 & 92.4 \\
\hline 3-M1 & 2.75 & 296 & $N$-dealkylation & - & - & - & 1.5 \\
\hline 3-M2 & 4.29 & 402 & Aromatic hydroxylation & - & - & - & 1.6 \\
\hline 3-M3 & 3.79 & 402 & Aromatic hydroxylation & - & - & - & 0.3 \\
\hline 3-M4 & 3.62 & 402 & Aromatic hydroxylation & - & 0.6 & 4.6 & 4.2 \\
\hline 4 & 5.19 & 441 & Parental compound & 100.0 & 47.0 & 33.3 & 12.4 \\
\hline 4-M1 & 3.37 & 457 & Aromatic hydroxylation & - & 53.0 & 66.6 & 87.6 \\
\hline
\end{tabular}

$t_{r}$ retention time 


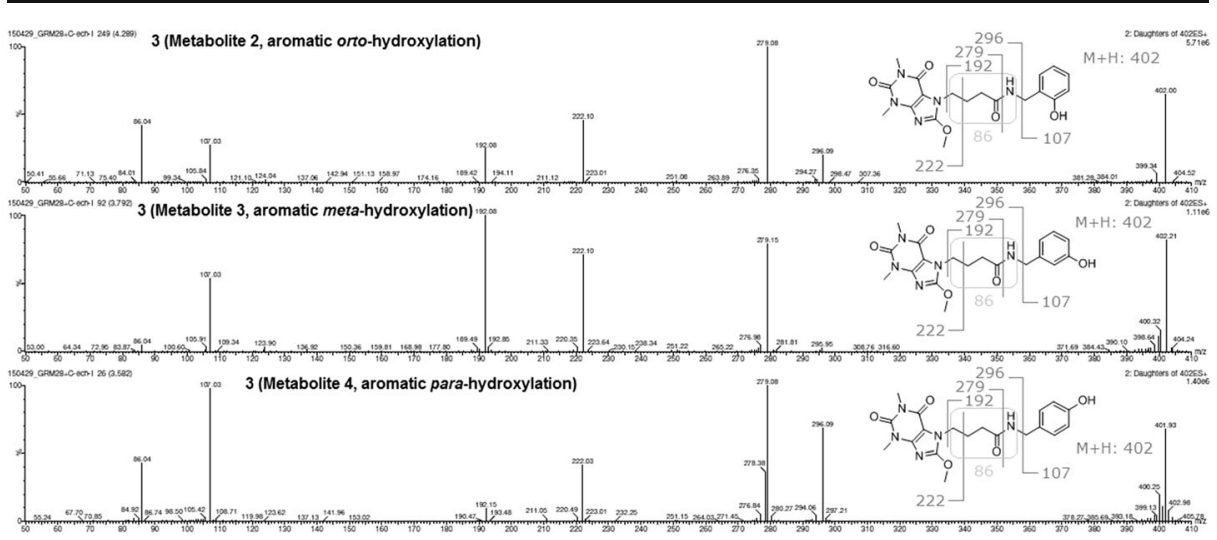

Fig. 3 LC-MS/MS spectra of compound 3 and its metabolites 3-M2, 3-M3, and 3-M4

parental compound (Fig. 4). Calculated in vitro half-time ( $\left.t_{1 / 2}\right)$ for 3 was $17.7 \mathrm{~min}$, and intrinsic clearance $\left(\mathrm{Cl}_{\text {int }}\right)$ was $97.88 \mu \mathrm{L} / \mathrm{min} / \mathrm{mg}$ protein (Fig. 5).

\section{Mutagenicity}

The results of mutagenicity assay in four Vibrio harveyi strains are shown in Table 3. None of the evaluated four 8-methoxy-purine-2,6-dione derivatives displayed mutagenicity at the concentration of $40 \mathrm{ng} / \mathrm{mL}$, as MI of four investigated compounds was always below $2.0 \mathrm{in}$

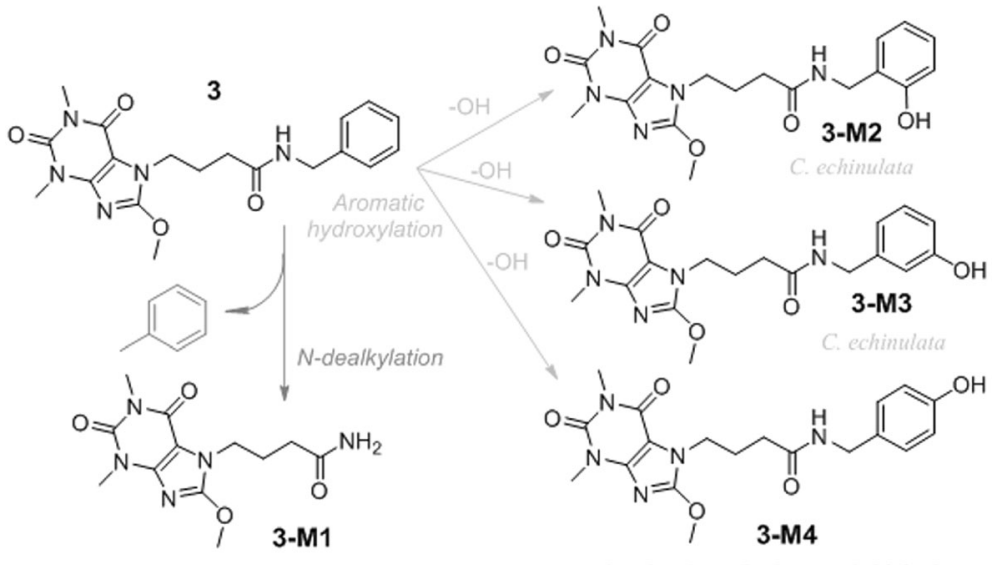

Microsomes, C. echimulata

\section{4-M1}

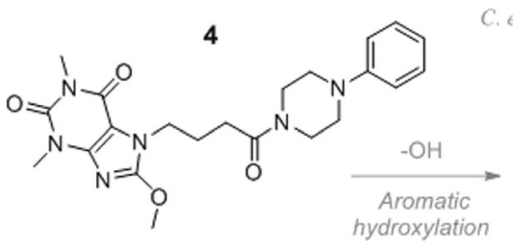<smiles>COc1nc2c(c(=O)n(C)c(=O)n2C)n1CCCC(=O)N1CCN(c2ccc(O)cc2)CC1</smiles>

Fig. 4 General scheme for compounds 3 and 4 biotransformation pathways in Cunninghamella and microsomal models 
Table 2 Metabolites of compound 3 observed in rat microsomal biotransformation after 30 min of incubation

\begin{tabular}{lllll}
\hline Cmp. & $t_{\mathrm{r}}(\mathrm{min})$ & {$[\mathrm{M}+\mathrm{H}]^{+}$} & Biotransformation reaction type & \% content among metabolites \\
\hline 3 & 4.61 & 386 & Parental compound & - \\
3 -M1 & 2.75 & 296 & $N$-dealkylation & 100 \\
\hline
\end{tabular}

$t_{r}$ retention time

all the Vibrio harveyi strains analyzed and the number of revertants counted in test samples was always lower than the values determined for NQNO controls. Moreover, all investigated compounds were also devoid of mutagenic activity according to the Ames test performed at the compound concentrations of 40 and $500 \mathrm{ng} / \mathrm{mL}$ (Table 3) using the Salmonella typhimurium TA100 strain.

\section{Antimutagenicity}

According to data obtained in the antimutagenicity assay (Table 4), four 8-methoxy-purine2,6-dione derivatives inhibited the mutagenic action of a standard mutagen NQNO in four Vibrio harveyi strains evaluated. All derivatives exhibited a very strong chemopreventive activity in two modified $V$. harveyi strains (BB7X and BB7M). The antimutagenic percentages calculated ranged from 56 to $74 \%$, and from 39 to $54 \%$ in BB7X and BB7M strains, respectively. In contrast, $1(20 \%), 2(38 \%)$, and $3(29 \%)$ exerted a moderate or a close to moderate chemopreventive effect in a wild BB7 strain, whereas $4(53 \%)$ was a strong chemopreventive agent in the same strain. Finally, chemopreventive activity exerted by the evaluated 8-methoxy-purine-2,6-dione derivatives was much lower in the BB7XM strain, in which chemopreventive percentages ranged from 9 to $15 \%$. Moreover, all derivatives showed a very potent antimutagenic activity in $S$. typhimurium TA100 strain. The chemopreventive percentages ranged from 88 to $96 \%$ and from 88 to $95 \%$ for concentrations of 40 and of $500 \mathrm{ng} / \mathrm{mL}$, respectively (Table 4).

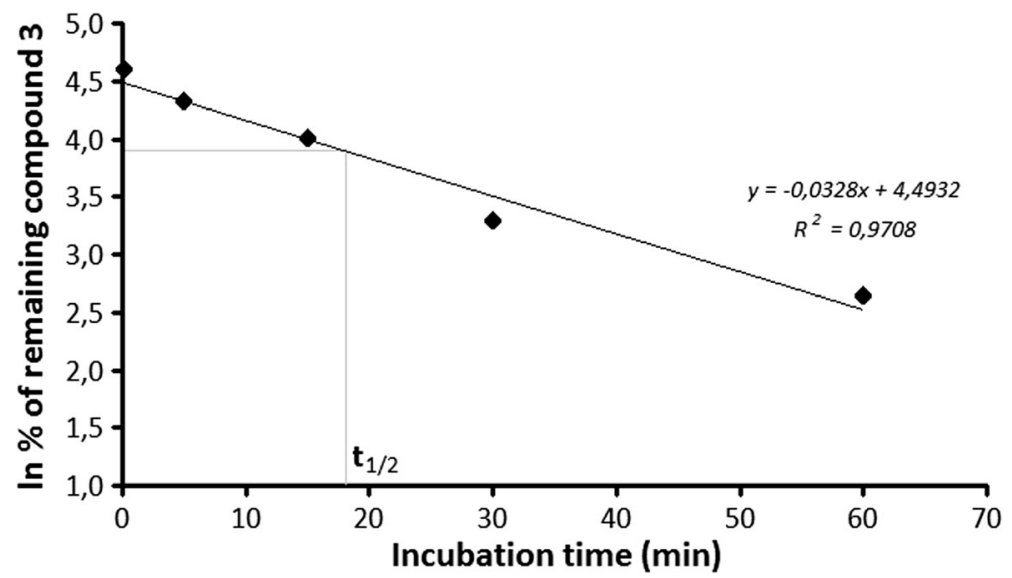

Fig. 5 Graphical calculation of $t_{1 / 2}$ during biotransformation in rat liver microsomes of compound 3 and its depletion along time 


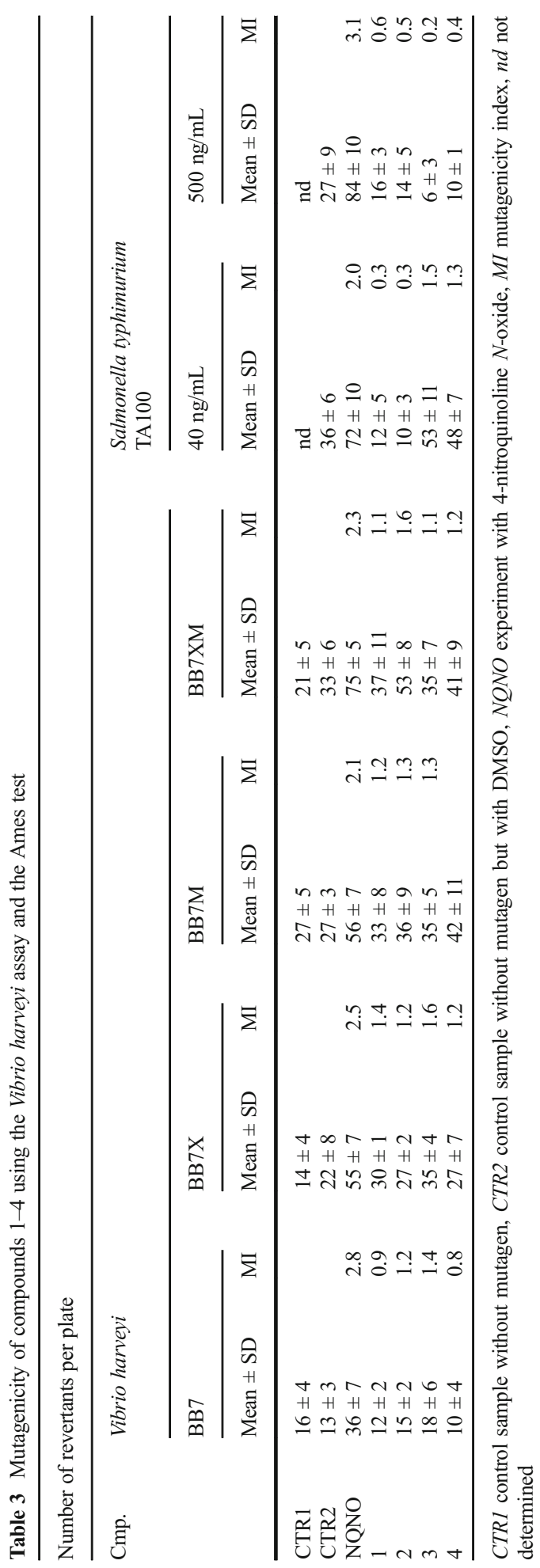




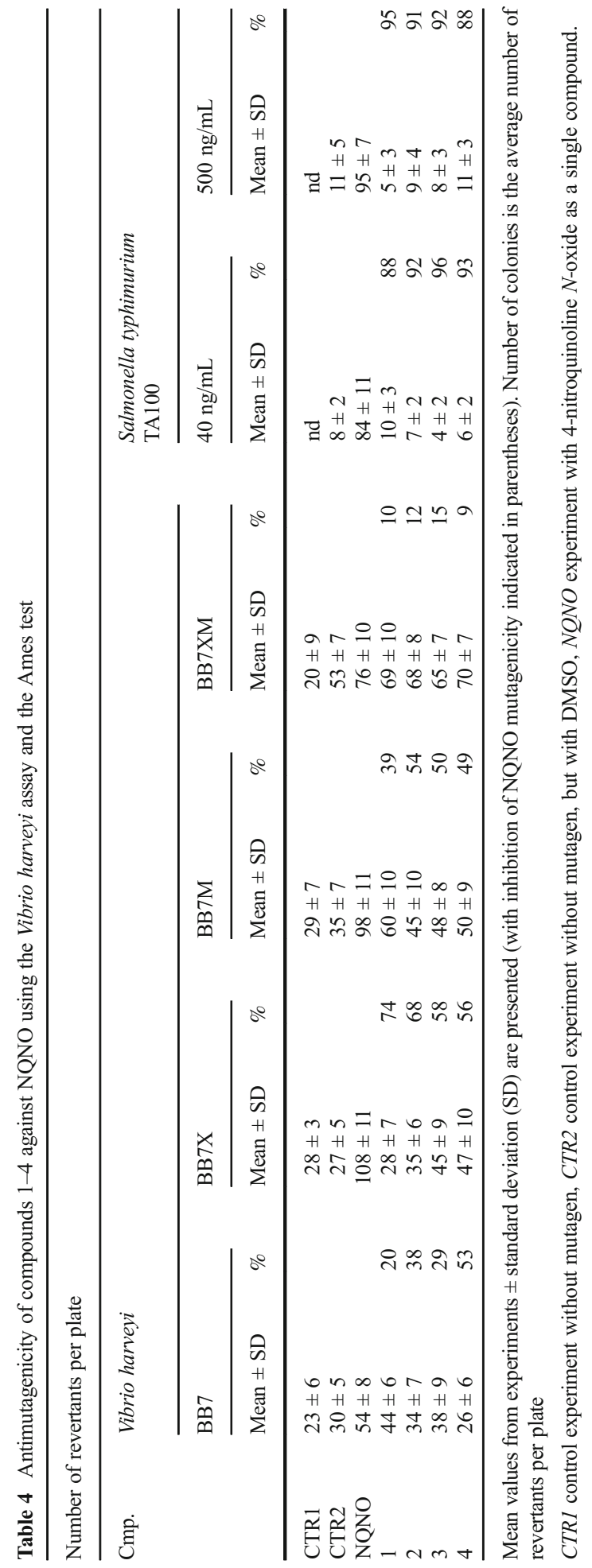




\section{Antioxidant Activity}

None of the compounds 1-4 showed capacity to scavenge DPPH radical, in comparison to positive controls considered (ascorbic and gallic acids). The maximum radical scavenging percentage observed was $6.56 \%$ for 3 at the concentration of $250 \mu \mathrm{M}$.

\section{Discussion}

Within the present study, in vitro biotransformation of some promising 8-methoxy-purine-2,6dione derivatives (1-4) was investigated. Additionally, muta-, antimutagenicity, and antioxidant potency of investigated compounds were assessed.

Among the tested compounds, 1 and 2 were metabolically stable as 1 did not undergo biotransformation in rat microsomes and no metabolites of 2 were detected in the Cunninghamella model. Cunninghamella biotransformation of compounds 3 and 4 rendered as metabolites the product of aromatic hydroxylation in both compounds and $\mathrm{N}$-dealkylation metabolite of compound 3. Nevertheless, this last metabolite was only detected in 3 with C. echinulata. With reference to M2 and M3 metabolites of 3, we have three possible different aromatic hydroxylation isomers: ortho-, meta- and para-hydroxylated metabolites. From a chemical point of view, $-\mathrm{CH}_{2}-\mathrm{NH}-\mathrm{R}$ substituent present in 4 is a mild electron donating moiety which activates slightly benzene ring in electrophilic aromatic substitution. In this reaction, the added hydroxyl group is directed preferably towards para- and ortho-positions, being meta-position the least favored. Furthermore, the steric hindrance plays an important role, promoting para- direction over ortho-direction. Therefore, considering the percentages of metabolites and their polarity (in reverse order than retention time, and supposing that parawill be slightly more polar than meta-, and meta- than ortho-), we hypothesize that the biotransformation products at retention times of 3.62, 3.79, and $4.29 \mathrm{~min}$ are the products of para-hydroxylation (3-M4), meta-hydroxylation (3-M3), and ortho-hydroxylation (3-M2) reactions of 3 , respectively. This hypothesis also explains why para-hydroxylated metabolite (3-M4) is the only one observed after the biotransformation of 3 in C. elegans and in C. blakesleeana. Regarding compound 4, by analogy with 3, we hypothesize that the product of its aromatic hydroxylation is para-hydroxylated derivative.

On the other hand, $N$-dealkylation product (3-M1) was a unique metabolite observed in microsomal biotransformation of 3 , being its intrinsic clearance quite high. Consequently, 8 methoxy-purine-2,6-dione ring is the most stable part of the molecule as it does not undergo biotransformations, which are limited to 7-alkyl substituent of purine core. Probably, compounds 1 and 2 do not suffer any biotransformation because they do not contain any aromatic ring or secondary amides like derivatives 3 and 4 .

Calculated in vitro half-time for 3 was $17.7 \mathrm{~min}$, and intrinsic clearance was $97.88 \mu \mathrm{L} / \mathrm{min} /$ $\mathrm{mg}$ protein. This $\mathrm{Cl}_{\text {int }}$ is high as it is 3.09 -fold lower than the $302.00 \mu \mathrm{L} / \mathrm{min} / \mathrm{mg}$ established for imipramine [23]. This experimental fact means that 3 is metabolized relatively fast.

Four 8-methoxy-purine-2,6-dione derivatives evaluated in this study are safe from a mutagenic point of view, as they successfully overcome two initial screenings of their mutagenic potential (the Vibrio harveyi and the Ames tests) without displaying mutagenic activity. Interestingly, all the investigated compounds showed antimutagenic potential, which means that they have chemopreventive activity. Therefore, they might potentially delay, inhibit, or even reverse carcinogenesis [38]. Nevertheless, the mechanism that explains their chemopreventive action is not the free radical 
scavenging activity, as they showed a low or non-existent capacity to quench DPPH radical in DPPH assay performed. Thus, further research should be performed in the future to ascertain the exact mechanism responsible for this chemopreventive activity [43, 44].

To sum up, within the study, metabolic profile and selected biological properties of some novel 8-methoxy-purine-2,6-dione derivatives (compounds 1-4) with analgesic and antiinflammatory properties were evaluated. It was demonstrated that in the Cunninghamella model, compound 2 did not undergo any biotransformation; whereas 3 and 4 showed less metabolic stability as they underwent biotransformation reactions. Moreover, the new derivatives of purine-2,6-dione do not possess mutagenic potential in the microbiological models considered and showed a strong chemopreventive activity in the modified Vibrio harveyi strains BB7X and BB7M.

Therefore, the obtained results might represent an important step in designing and planning future studies with purinediones.

Acknowledgements This work was supported by Jagiellonian University Medical College under Grants $\mathrm{K} / \mathrm{DSC} / 002885$ and $\mathrm{K} / \mathrm{ZDS} / 005488$.

\section{Compliance with Ethical Standards}

Conflict of Interest The authors declare that they have no conflict of interest.

Open Access This article is distributed under the terms of the Creative Commons Attribution 4.0 International License (http://creativecommons.org/licenses/by/4.0/), which permits unrestricted use, distribution, and reproduction in any medium, provided you give appropriate credit to the original author(s) and the source, provide a link to the Creative Commons license, and indicate if changes were made.

\section{References}

1. Asha, S., \& Vidayavathi, M. (2009). Cunninghamella - a microbial model for drug metabolism studies - a review. Biotechnology Advances, 27, 16-29.

2. Amadio, J., \& Murphy, C. D. (2011). Production of human metabolites of the anti-cancer drug flutamide via biotransformation in Cunninghamella species. Biotechnology Letters, 33, 321-326.

3. Pękala, E., Kubowicz, P., \& Łażewska, D. (2012). Cunninghamella as a microbiological model for metabolism of histamine H3 receptor antagonist 1-[3-(4-tert butylphenoxy)propyl]piperidine. Applied Biochemistry and Biotechnology, 168, 1584-1593.

4. Watanabe, S., Kuzhiumparambil, U., Winiarski, Z., \& Fu, S. (2016). Biotransformation of synthetic cannabinoids JWH-018, JWH-073 and AM2201 by Cunninghamella elegans. Forensic Science International, 261, 33-42.

5. Pękala, E., Kochan, M., \& Carnell, A. J. (2009). Biotransformation of synthetic cannabinoids JWH-018, JWH-073 and AM2201 by Cunninghamella elegans. Letters in Applied Microbiology, 48, 19-24.

6. Piska, K., Żelaszczyk, D., Jamrozik, M., Kubowicz-Kwaśny, P., \& Pękala, E. (2016). Cunninghamella biotransformation - similarities to human drug metabolism and its relevance for the drug discovery process. Current Drug Metabolism, 17, 107-117.

7. Srisailam, K., Raj Kumar, V., \& Veeresham, C. (2010). Predicting drug interaction of Clopidogrel on microbial metabolism of diclofenac. Applied Biochemistry and Biotechnology, 160, 1508-1516.

8. Srisailam, K., \& Veeresham, C. (2010). Biotransformation of celecoxib using microbial cultures. Applied Biochemistry and Biotechnology, 160, 2075-2089.

9. Amadio, J., Gordon, K., \& Murphy, C. D. (2010). Biotransformation of flurbiprofen by Cunninghamella species. Applied and Environmental Microbiology, 76, 6299-6303.

10. Bourdon, F., Lecoeur, M., Verones, V., Vaccher, C., Lebegue, N., Dine, T., Kambia, N., \& Goossens, J. F. (2013). In vitro pharmacokinetic profile of a benzopyridooxathiazepine derivative using rat microsomes and hepatocytes: identification of phases I and II metabolites. J. Pharmaceut. Biomed., 80, 69-78. 
11. Ahn, S., Kearbey, J. D., Li, C. M., Duke 3rd, C. B., Miller, D. D., \& Dalton, J. T. (2011). Biotransformation of a novel antimitotic agent, I-387, by mouse, rat, dog, monkey, and human liver microsomes and in vivo pharmacokinetics in mice. Drug Metabolism and Disposition, 39, 636-643.

12. Sun, H. (2012). Capture hydrolysis signals in the microsomal stability assay: molecular mechanisms of the alkyl ester drug and prodrug metabolism. Bioorganic \& Medicinal Chemistry Letters, 22, 989-995.

13. Marques, L. M., da Silva Jr., E. A., Gouvea, D. R., Vessecchi, R., Pupo, M. T., Lopes, N. P., Kato, M. J., \& de Oliveira, A. R. (2014). In vitro metabolism of the alkaloid piplartine by rat liver microsomes. Journal of Pharmaceutical and Biomedical Analysis, 95, 113-120.

14. Asha, S., \& Vidyavathi, M. (2010). Role of human liver microsomes in in vitro metabolism of drugs-a review. Applied Biochemistry and Biotechnology, 160, 1699-1722.

15. Zygmunt, M., Chłon-Rzepa, G., \& Sapa, J. (2014). Analgesic and anti-inflammatory activity of 7substituted purine-2,6-diones. Pharm. Rep., 66, 996-1002.

16. Zygmunt, M., Chłon-Rzepa, G., Sapa, J., \& Pawłowski, M. (2015). Analgesic activity of new 8-methoxy-1, 3-dimethyl-2,6-dioxo-purin-7-yl derivatives with carboxylic, ester or amide moieties. Pharm. Rep., 67, 9-16.

17. Quinn, L., Dempsey, R., Casey, E., Kane, A., \& Murphy, C. D. (2015). Production of drug metabolites by immobilised Cunninghamella elegans: from screening to scale up. Journal of Industrial Microbiology \& Biotechnology, 42, 799-806.

18. Hamelin, B. A., Bouayad, A., Drolet, B., Gravel, A., \& Turgeon, J. (1998). In vitro characterization of cytochrome P450 2D6 inhibition by classic histamine H1 receptor antagonists. Drug Metabolism and Disposition, 26, 536-539.

19. Huang, J., Si, L., Fan, Z., Hu, L., Qiu, J., \& Li, G. (2011). In vitro metabolic stability and metabolite profiling of TJ0711 hydrochloride, a newly developed vasodilatory $\beta$-blocker, using a liquid chromatography-tandem mass spectrometry method. Journal of Chromatography. B, Analytical Technologies in the Biomedical and Life Sciences, 879, 3386-3392.

20. Di, L., Kerns, E. H., Hong, Y., Kleintop, T. A., McConnell, O. J., \& Huryn, D. M. (2003). Optimization of a higher throughput microsomal stability screening assay for profiling drug discovery candidates. Journal of Biomolecular Screening, 8, 453-462.

21. Canale, V., Kurczab, R., Partyka, A., Satała, G., Słoczyńska, K., Kos, T., Jastrzębska-Więsek, M., Siwek, A., Pękala, E., Bojarski, A. J., Wesołowska, A., Popik, P., \& Zajdel, P. (2016). $N$-alkylated arylsulfonamides of (aryloxy)ethyl piperidines: 5-HT7 receptor selectivity versus multireceptor profile. Bioorganic \& Medicinal Chemistry, 24, 130-139.

22. Słoczyńska, K., Pańczyk, K., Waszkielewicz, A. M., Marona, H., \& Pękala, E. (2016). In vitro mutagenic, antimutagenic and antioxidant activities evaluation and biotransformation of some bioactive 4-substituted 1(2-methoxyphenyl)piperazine derivatives. Journal of Biochemical and Molecular Toxicology, 30, 593-601.

23. Singh, J. K., Solanki, A., \& Shirsath, V. S. (2012). Comparative in vitro intrinsic clearance of imipramine in multiple species liver micrososmes: human, rat, mouse and dog. J. Drug Metab. Toxicol., 3, 126.

24. Basavapathruni, A., Olhava, E. J., Daigle, S. R., Therkelsen, C. A., Jin, L., Boriack-Sjodin, P. A., Allain, C. J., Klaus, C. R., Raimondi, A., Scott, M. P., Dovletoglou, A., Richon, V. M., Pollock, R. M., Copeland, R. A., Moyer, M. P., Chesworth, R., Pearson, P. G., \& Waters, N. J. (2014). Nonclinical pharmacokinetics and metabolism of EPZ-5676, a novel DOT1L histone methyltransferase inhibitor. Biopharmaceutics \& Drug Disposition, 35, 237-252.

25. Czyż, A., Jasiecki, J., Bogdan, A., Szpilewska, H., \& Węgrzyn, G. (2000). Genetically modified Vibrio harveyi strains as potential bioindicators of mutagenic pollution of marine environments. Applied and Environmental Microbiology, 66, 599-605.

26. Piosik, J., Ulanowska, K., Gwizdek-Wiśniewska, A., Czyż, A., Kapuściński, J., \& Węrzyn, G. (2003). Alleviation of mutagenic effects of polycyclic aromatic agents (quinacrine mustard, ICR-191 and ICR-170) by caffeine and pentoxifylline. Mutation Research, 530, 47-57.

27. Podgórska, B., Chęć, E., Ulanowska, K., \& Wegrzyn, G. (2005). Optimisation of the microbiological mutagenicity assay based on genetically modified Vibrio harveyi strains. Journal of Applied Genetics, 46, 241-246.

28. Podgórska, B., \& Wegrzyn, G. (2006). A modified Vibrio harveyi mutagenicity assay based on bioluminescence induction. Letters in Applied Microbiology, 42, 578-582.

29. Mortelmans, K., \& Zeiger, E. (2000). The Ames Salmonella/microsome mutagenicity assay. Mutation Research, 455, 29-60.

30. Zeiger, E. (2013). Bacterial mutation assays. Methods in Molecular Biology, 1044, 3-26.

31. Gulluce, M., Agar, G., Baris, O., Karadayi, M., Orhan, F., \& Sahin, F. (2010). Mutagenic and antimutagenic effects of hexane extract of some Astragalus species grown in the eastern Anatolia region Turkey. Phytotherapy Research, 24, 1014-1018.

32. Fronza, G., Campomenosi, P., Iannone, R., \& Abbondandolo, A. (1992). The 4-nitroquinoline 1-oxide mutational spectrum in single stranded DNA is characterized by guanine to pyrimidine transversions. Nucleic Acids Research, 20, 1283-1287. 
33. Powroźnik, B., Słoczyńska, K., Canale, V., Grychowska, K., Zajdel, P., \& Pękala, E. (2016). Preliminary mutagenicity and genotoxicity evaluation of selected arylsulfonamide derivatives of (aryloxy)alkylamines with potential psychotropic properties. Journal of Applied Genetics, 57, 263-270.

34. Słoczyńska, K., Waszkielewicz, A. M., \& Marona, H. (2014). Preliminary assessment of mutagenic and anti-mutagenic potential of some aminoalkanolic derivatives of xanthone by use of the Vibrio harveyi assay. Mutation Research, 768, 8-13.

35. Słoczyńska, K., Pękala, E., Wajda, A., Węgrzyn, G., \& Marona, H. (2010). Evaluation of mutagenic and antimutagenic properties of some bioactive xanthone derivatives using Vibrio harveyi test. Letters in Applied Microbiology, 50, 252-257.

36. Pękala, E., Liana, P., Kubowicz, P., Powroźnik, B., Obniska, J., Chlebek, I., Węgrzyn, A., \& Węgrzyn, G. (2013). Evaluation of mutagenic and antimutagenic properties of new derivatives of pyrrolidine-2,5-dione with anti-epileptic activity, by use of the Vibrio harveyi mutagenicity test. Mutation Research, 758, 18-22.

37. Kamiński, K., Obniska, J., Chlebek, I., Liana, P., \& Pękala, E. (2013). Synthesis and biological properties of new $\mathrm{N}$-Mannich bases derived from 3-methyl-3-phenyl- and 3,3-dimethyl-succinimides. Part $V$. Eur. J. Med. Chem., 66, 12-21.

38. Delarmelina, J. M., Dutra, J. C., \& Batittucci Mdo, C. (2014). Antimutagenic activity of ipriflavone against the DNA-damage induced by cyclophosphamide in mice. Food and Chemical Toxicology, 65, 140-146.

39. Domínguez-Álvarez, E., Plano, D., Font, M., Calvo, A., Prior, C., Jacob, C., Palop, J. A., \& Sanmartin, C. (2014). Synthesis and antiproliferative activity of novel selenoester derivatives. European Journal of Medicinal Chemistry, 73, 153-166.

40. Martins, M., Arantes, S., Candeias, F., Tinoco, M. T., \& Cruz-Morais, J. (2014). Antioxidant, antimicrobial and toxicological properties of Schinus molle L. essential oils. Journal of Ethnopharmacology, 151, 485492.

41. Arora, D. S., \& Chandra, P. (2011). In vitro antioxidant potential of some soil fungi: screening of functional compounds and their purification from Penicillium citrinum. Applied Biochemistry and Biotechnology, 165, 639-651.

42. Tarhan, L., Nakipoğlu, M., Kavakcioğlu, B., Tongul, B., \& Nalbantsoy, A. (2016). The induction of growth inhibition and apoptosis in HeLa and MCF-7 cells by Teucrinumsandrasicum, having effective antioxidant properties. Applied Biochemistry and Biotechnology, 178, 1028-1041.

43. Watanabe, M., Kobayashi, H., \& Ohta, T. (1994). Rapid inactivation of 3-chloro-4-(dichloromethyl)-5hydroxy-2(5H)-furanone (MX), a potent mutagen in chlorinated drinking water, by sulfhydryl compounds. Mutation Research, 312, 131-138.

44. Słoczyńska, K., Powroźnik, B., Pękala, E., \& Waszkielewicz, A. M. (2014). Antimutagenic compounds and their possible mechanisms of action. Journal of Applied Genetics, 55, 273-285. 